EPiC Series in Computing
Volume 78, 2021, Pages 22-30
Proceedings of the European Univer-
sity Information Systems Conference 2021

\title{
Joint Forces: Merging Competencies and Cultures in University IT
}

\author{
Dominik Rudolph ${ }^{1}$, Anne Thoring ${ }^{1}$, Raimund Vogl ${ }^{1}$, Alice Fleury ${ }^{1}$ \\ ${ }^{1}$ University of Münster, Germany \\ d.rudolph@wwu.de, a.thoring@wwu.de, rvogl@wwu.de, \\ alice.fleury@wwu.de
}

\begin{abstract}
Mergers in university IT are a major challenge, as ongoing operations should be affected as little as possible. At the same time, it is not uncommon for different work cultures to collide between data centres, decentralized IT and administrative IT. There are also different expectations on the part of the user groups that need to be met. The merger of the Zentrum für Informationsverarbeitung (the IT centre with approx. 100 employees whose focus is on infrastructure) and the Stabsstelle IT und Prozessentwicklung (the administrative IT department with approx. 60 employees whose focus is on operations and organisation projects) to form the "WWU IT", which took place at Münster University (WWU) at the beginning of 2020, can serve as a good example for similar IT restructuring at universities.
\end{abstract}

\section{Introduction}

While mergers with other organisations and restructuring within an organisation take place regularly in the business sector, German universities often shy away from this step. One reason for this could be that the advantages are not as obvious, as monetary synergy gains in terms of staff savings are not immediately realisable in the German public sector. Nevertheless, especially in university IT, there can be great advantages of an efficient, merged organisation that counteract possible disadvantages and justify the reorganisation efforts. Typically, it is large universities that have a data centre for the provision of infrastructure, an IT for the administration as well as decentralised IT units in the departments. This differentiation and division of responsibilities dates back to the 1980s and 1990s, when it made sense to increasingly specialise IT to meet a wide variety of needs. However, the advantages of specialisation are disappearing due to the general standardisation of hardware and software and are now opposed by significant disadvantages: On the one hand, the traditional division between the IT centre and the administrative IT is becoming increasingly blurred due to the expanded range of services emerging from digitalization, with the result that there is a risk of redundant work and a lack of transparency for users caused by unclear responsibilities. On the other hand, the complex needs 
of current IT projects require powerful large units with sufficient human and financial resources that small units competing with each other for internal resources could not provide. Against this background, Münster University decided to merge the previously independent units Zentrum für Informationsverarbeitung (IT centre) and Stabsstelle IT und Prozessentwicklung (administrative IT) into the new unit "WWU IT" with over 160 employees as of 01.01.2020.

\section{Implementation Strategy}

Following Jansen (2004, pp. 150-152), Picot \& Picot (2012, p. 26) and Luik (2014, p. 10), a merger process can be divided into three phases: 1) the pre-merger phase, focusing on the strategic development of a vision in light of the status quo, 2) the merger phase, focusing on the planning and implementation of the organisational merger, and 3) the post-merger phase, focusing on the integration of the organisational cultures and the customer acceptance. These phases are not to be understood as rigidly consecutive with regard to the associated tasks, but represent the respective task focus and milestones that must be fulfilled at a certain point in the process to ensure the success of the merger. Table 1 shows the tasks that were assigned to the individual phases of the WWU IT merger. They will be described in detail in the following chapters.

\begin{tabular}{|c|c|c|}
\hline Pre-Merger & Merger & Post-Merger \\
\hline $\begin{array}{l}\text { - } \\
\text { - } \\
\text { - } \\
\text { Development of a vision } \\
\text { - } \quad \text { Demmunication strategy } \\
\text { integration strategy } \\
\text { - } \\
\text { Relationship management }\end{array}$ & $\begin{array}{l}\text { Reorganisation of the } \\
\text { organisational and } \\
\text { operational structure } \\
\text { - } \quad \begin{array}{l}\text { Development of a } \\
\text { common identity }\end{array} \\
\text { - Internal communication } \\
\text { measures } \\
\text { - Personnel measures }\end{array}$ & $\begin{array}{ll}\text { - } & \text { Integration of cultures } \\
\text { - } & \text { Acceptance management } \\
\text { - } & \text { Stakeholder relations } \\
\text { - } & \text { Review of implementation }\end{array}$ \\
\hline
\end{tabular}

Table 1: Tasks by merger phase

\subsection{Pre-Merger Phase: Vision}

Against the background of the developments in the IT and higher education sectors briefly discussed above, it is necessary to formulate a clear vision of the new unit "WWU IT". In addition, the concrete objectives of the merger must be defined, as this will not only ensure that the implementation process is coordinated and goal-oriented, but also create an argumentative basis for the communication with two vital stakeholder groups: employees and customers. With a convincing long-term goal in mind, it is more likely that employees will understand and accept necessary interventions in their working environment, and customers will tolerate temporary inconveniences caused by the merger.

The vision for WWU IT - which was revised during the merger in exchange with the employees is intended as an ideal that should also guide the daily work at the unit. It is therefore part of the mission statement. The vision describes the new unit in the following way:

WWU IT is the service and competence centre for reliable, customer-oriented and sustainable IT at Münster University. With its services, it supports all WWU members in the areas of study, teaching, research and administration. Their requirements and feedback significantly guide its strategy and actions. As an academic institution, WWU IT actively participates in research and teaching and lives a 
culture of independent knowledge acquisition and work. Above all, WWU IT strives to make Münster University a role model for university digitisation.

WWU IT works together with all divisions of Münster University in a solution-oriented and collegial manner and promotes digital key competences. In particular, WWU IT is part of the university's cooperative IT supply system and is thus a cooperation partner of the decentral IT support units, the University and State Library of Münster, the communication and public relations department and the administrative departments. Beyond this, WWU IT plays a leading role in state-wide cooperations and is involved at national and international level. WWU IT is networking with other university IT operators and embedded in cooperations and committees.

The specific objectives of the merger serve this overarching vision and can be summarised into two main goals: First, the merger should tap synergies which improve the IT support for study, teaching, research, and administration. Second, it should create a more powerful and efficient organisation with regard to current and future major projects (e.g. introduction of a new campus management system, migration of the ERP system, introduction of a research data infrastructure, etc.). To achieve these objectives, an implementation strategy was developed.

\subsection{Merger Phase: Structure \& Identity}

With the support of an external consulting firm, the merger phase was initiated at the end of 2019 with a staff meeting and completed in March 2021. It was characterised by the following focal points:

- creation of organisational structures,

- development of synergies (cross cutting topics),

- identification of optimisation potential (stakeholder interviews), and

- creation of a common identity.

In order to create organisational structures, the structures of university IT units at other universities in Germany and abroad were first surveyed. As it turned out, these were very heterogeneous and often seemed to have grown historically rather than being organised according to aspects that were comprehensible from an external perspective. Subsequently, various organisational structures were developed and evaluated internally - with the result that a large-scale reorganisation did not have sufficiently clear advantages to justify the significant impact of such a measure on the functioning of IT in the form of frictions in established processes and a reduction in staff satisfaction.

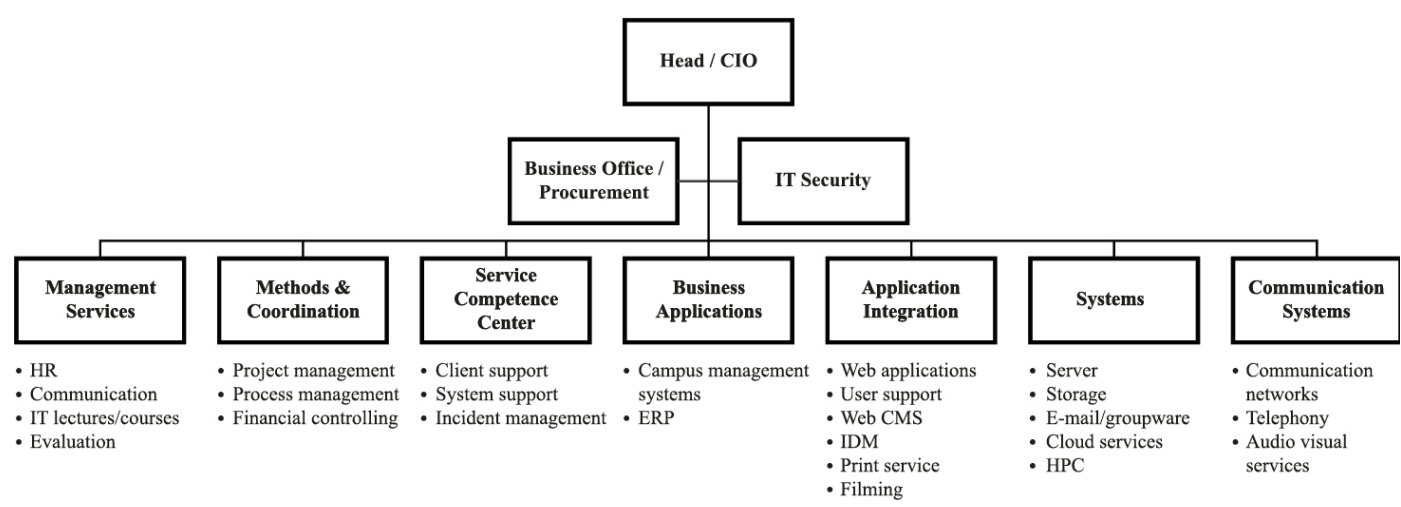

Figure 1: WWU IT organisational structure 
As a consequence, a conservative structure was chosen in which the previous units (four from the IT centre, three from administrative IT) were left largely unchanged and placed under a common management as seven equal departments, with the heads of department building a joined management board (Figure 1). Three of the units were already formed as departments. The other units were working groups that were upgraded to departments as part of the merger. In the course of staffing the management level, the issue of gender equality was given high importance. The position of deputy head of WWU IT, which was filled twice for reasons of distinctive competencies brought in by the two institutions, could be filled in a gender-equitable manner. In addition, the departments are now managed by a total of 2 female heads and 5 male heads as well as 5 female deputy heads and 2 male deputy heads. Especially for the IT centre, this is a significant improvement as there were previously no female (deputy) heads of department. The chosen organisational structure proved to be very effective and appropriate in the course of the year, even during the massive challenges of the Corona pandemic. Despite the small overlaps, the employees and their management succeeded in developing pragmatic and consensual work solutions.

Cross-cutting topics are a good starting point for developing synergies, as a particularly high synergy potential can be expected here due to similar topics. Here the employees' shared competences create a basis for discussion at eye level; at the same time, the different experiences are an impetus for discourse and improvement. Three cross cutting topics were identified at WWU IT: business office processes (especially procurement), client operations and service processes. In various workshops, working methods, responsibilities and processes were identified together with the employees of the departments concerned, thus laying the foundation for improved cooperation in the future. During the workshops, important learnings also emerged as a result of the better insight into the topic areas. For example, because of the very different customer groups that the service team supports, the original idea of "one face to the customer" was abandoned and the creation of two customer group focused hotlines with interfaces between them was defined as a new goal.

Another important component of the merger phase was the identification of optimisation potential via evaluation of WWU IT by its stakeholders. For this purpose, the external consultants conducted extensive structured interviews with a total of 12 stakeholder representatives, which supplemented the already existing quantitative evaluation in the form of an annual user survey with a qualitative methodology. The expert group was formed to reflect the diversity of users and stakeholders of WWU

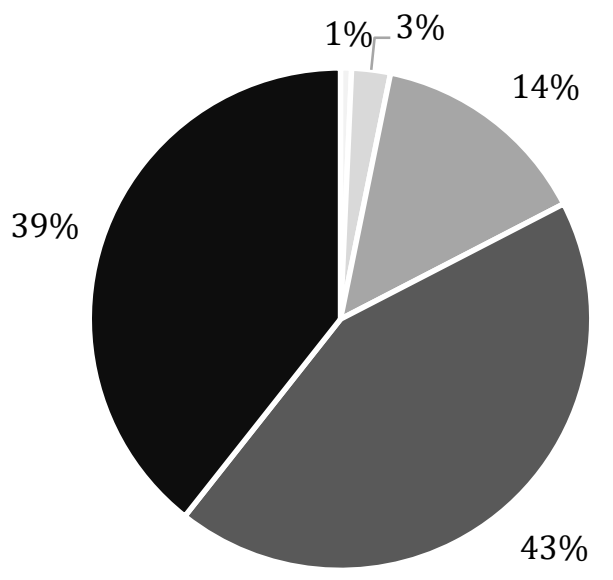

\author{
very bad \\ bad \\ partly/partly \\ - good \\ - very good
}

Figure 2: Evaluation of the Corona crisis management by WWU IT $(n=2,688)$ 
IT with their different - sometimes conflicting - needs and approaches. The expert interviews are intended to show whether adjustments to existing services or the creation of new services are necessary. On the one hand, they expand the understanding of the prevailing services, applications, processes, channels and structures, and on the other hand, they shed light on possible future refinements of WWU IT. Summarised to the essentials, both the service portfolio and the organisational structures of WWU IT appear to be very efficient and user-friendly in the external perception. Particularly in supporting stakeholders during the pandemic, which was very challenging for IT due to the need for short-term solutions in the areas of home office and digital teaching, WWU IT was able to convince with a strong performance from the customer's point of view and also build a reputation on this. The quantitative user survey that was conducted in autumn 2020 confirms this evaluation, as Figure 2 demonstrates.

The creation of a common identity was of particular importance in the merger because of the largely unchanged organisational structure, which provides only few points of contact between some of the departments. Previously identity-creating features such as name and logo were deliberately abandoned in favour of new ones in order to visually express a merger at eye level (instead of a takeover) despite the different sizes of the institutions. The employees were actively involved in finding the name and logo. In workshops moderated by external consultants, a mission statement was developed together with all employees, which describes the self-image and the way of working and cooperating with each other. This also revealed existing reservations, prejudices and problem areas that could subsequently be addressed. In addition, an anonymous staff survey was conducted at the beginning of the merger process in late 2019, and a likewise anonymous idea box was set up to create space for open feedback, honest assessments and employees' own ideas. Something that was positively surprising was that the majority of employees (66\%) expected the merger to be an opportunity and not as a risk - both personally and organisationally. Interestingly, employees of the smaller institution involved in the merger, the administrative IT, were the most optimistic (Figure 3).

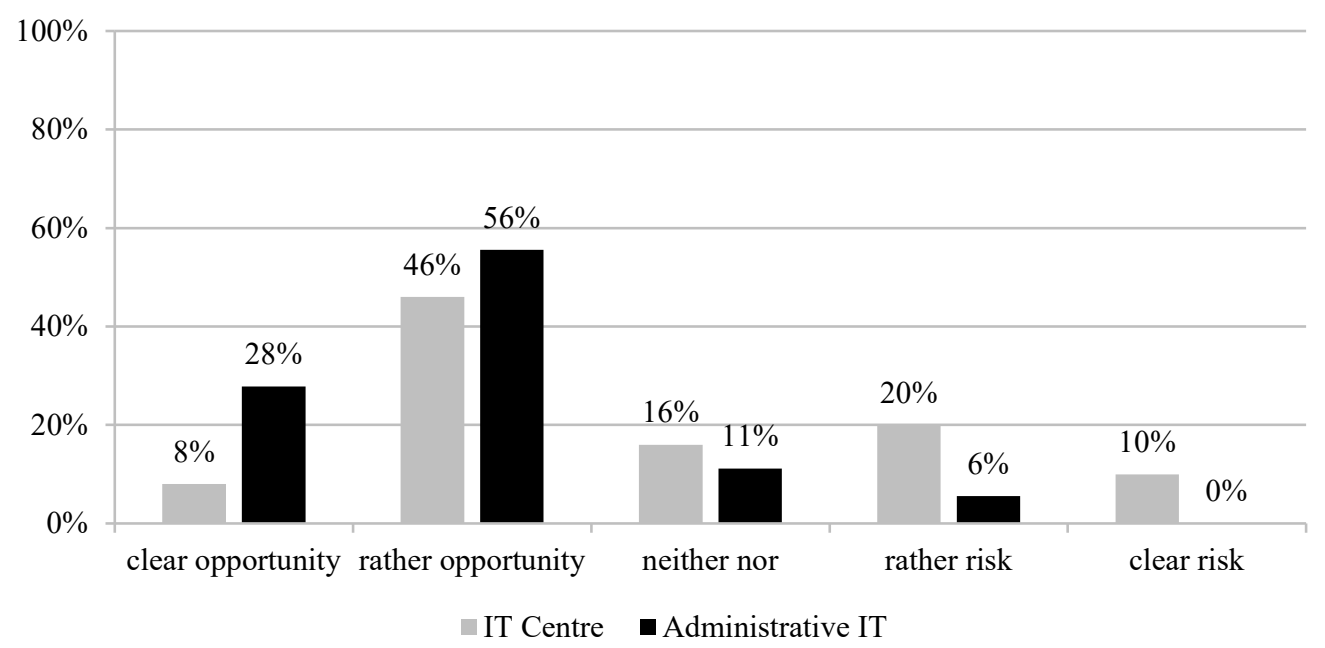

Figure 3: Employees' expectations of the merger by institution $\left(\mathrm{n}_{\mathrm{IT} \text { centre }}=50, \mathrm{n}_{\text {administrativelT }}=36\right)$

The open comments in the survey showed great interest and at the same time a general insecurity on the part of the employees - both of which lead to the desire for continuous information and as much transparency as possible from the management level. This is exemplified by the following comment from a participant: "Personal insecurity is felt by everyone and is a risk factor. Therefore, the most important thing is to send clear messages from the beginning and present personal implications of the 
merger to each individual". The responses also revealed the key hopes and concerns. As mentioned by another participants, "[t]he challenge will certainly be to bring together the sometimes very different structures, ways of working, customers, etc.", but at the same time, high hopes are placed in new colleagues and their differing approaches and perspectives to question, reform and revitalise existing structures and processes. For some, the official joining of forces also means overcoming a past where - in their perception - "there was always a certain competition, no sense of community. I hope that the team spirit will develop positively". In sum, the most important hopes include improvements in communication and collaboration as well as optimisations of organisational structures and work processes. In addition, opening up new opportunities for personal development has a high priority.

When it comes to concerns, employees primarily see the risk of "additional overtime with already heavy workloads, which is why dealing with the problem of understaffing in the face of increasing demands should take a central role". Some also expect frictions in the merger process. When it comes to customer satisfaction and image, especially IT centre employees fear negative: The IT centre "has a very good reputation and can only loose, but not benefit. If the name were to be changed to reflect the merger, many reputations built up over years would have to be rebuilt". As might be expected, concerns about the loss of responsibilities and competencies also play a significant role in the prevailing negative expectations.

One specific aspect mentioned particularly frequently in the responses was the sensitive issue of salaries, as many employees felt that there was an imbalance between the previous units, given that there were numerous scientific employees and civil servants in the IT centre, but almost none in the administrative IT unit. Such obstacles hinder the identification with the organisation and the social cohesion of the employees, therefore transparency had to be created and a short-term perspective for the review of the respective pay grade groupings had to be shown in order not to let this issue become a stumbling block for the formation of a common identity. All in all, however, the atmosphere can be described as optimistic and showed the employees' motivation to realise opportunities for improvement for Münster University, which should be appreciated as a good foundation.

\subsection{Post-Merger Phase: Culture}

In addition to the organisational integration, the cultural integration is crucial for the success of a merger. This is all the truer for WWU IT, as the organisational structures were largely retained and changes in this regard took place primarily at management level. Subsequently, the achievement of the merger goals is essentially based on the cooperation and cohesion of the employees, because the development of synergies and a more efficient work organisation mean above all a reorganisation of responsibilities beyond the predefined structures and an intensified exchange to benefit from the newly gained competences. This necessarily involves compromises to combine the best of both worlds.

At the organisational level, the foundation for a common organisational culture was laid by creating a common identity and formulating common values. However, in order for a shared organisational culture to actually emerge and be lived, communication and direct contact between the employees is needed above all. Only in this way can noticeable cultural differences be explained, understood and processed to ultimately trigger a learning process on both sides. Of all things, personal contact had unfortunately to be largely suspended during the central phase of the merger due to the Corona pandemic. Exchanges in common rooms and at summer and winter parties were eliminated. Accordingly, communication measures were important that not only counteract the increasing isolation of employees and maintain opportunities for informal exchange, but also make it possible for previously independent teams to get to know each other and, ideally, even grow together. In addition to established communication and evaluation formats such as employee-supervisor discussions and employee surveys, the following formats were generated for this purpose:

- fusion café, 
- open dialogue and open office hours, and

- shared internal wiki and group chat.

The Fusion Café simulates informal exchange in common rooms and was originally planned as a one-hour face-to-face event, but was predominantly implemented in digital form. The aim of the format was for employees to get to know each other across departmental boundaries and to get a better overview of the respective work areas and responsibilities. In order to reduce communication barriers, the events were topic-based (with a focus on customer communication, project management, home office, sustainable working in IT, among others) and selected colleagues provided insight into their experiences with the respective topic area at the beginning of the meetings as an opener for the exchange in small groups. The Fusion Café was explicitly not intended as a workshop and was attended and evaluated so well by employees that it has been continued as the "IT Café" since the beginning of 2021.

The management has offered the open dialogue format, which meets the employees' demand for more transparency and information regarding topics at management level. Here, the WWU IT management regularly reports to all interested employees on current projects and subsequently answers questions in an open discourse and accepts feedback and suggestions. The open dialogue is complemented by the open office hours, which employees can use as needed to have individual discussions with the management.

A shared internal wiki was set up via the Confluence platform, which is not only used for exchange and documentation within working groups, but also gives all employees an insight into the work tasks, responsibilities and projects of the respective other departments. Contact persons can thus be found more quickly and internal communication can be improved. The wiki has proven its worth and is used intensively by all departments. The same applies to channels in the group chat service Mattermost, which are used across departments and have established themselves as an informal, uncomplicated way of communication.

As in the previous year, the annual employee survey in 2020 focused on the merger, this time also with special attention to the development of the organisational culture and the measures taken in this regard. Staff feedback regarding communication and cooperation during the merger (Figure 4) shows that while a solid foundation has been laid, there is still significant room for improvement - a satisfactory result for now, given the challenges of the Corona pandemic in the year of the merger. The opportunities for participation in the merger process $(\overline{\mathrm{X}}=3.2)$ and the management's communication $(\overline{\mathrm{X}}=3.3)$ were rated just above average. The cooperation of the staff, despite far-reaching contact restrictions, is rated at least "satisfactory" to "good" $(\overline{\mathrm{X}}=3.5)$. The informal Fusion Café $(\overline{\mathrm{X}}=3.6)$ has the best value in comparison. It has gained relevance because it not only connects the staff of the two merging institutions on a social level, but also avoids alienating the employees who already know each other.

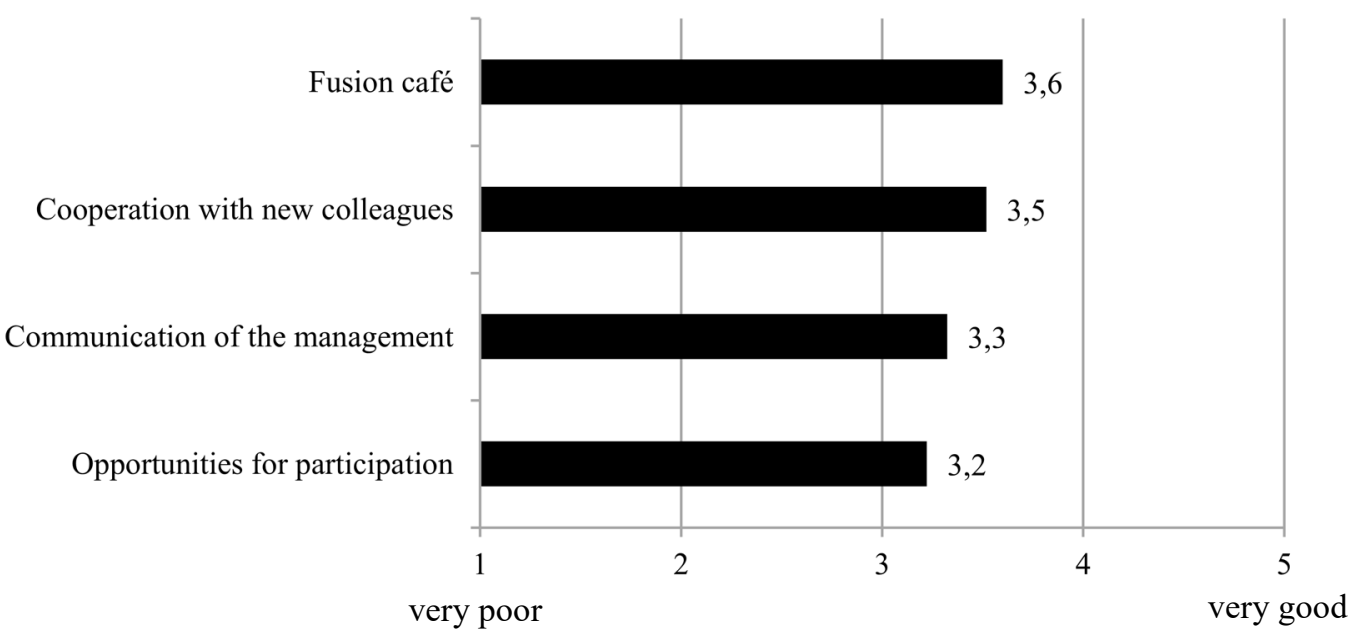

Figure 4: Employees' assessment of communication and cooperation, arithmetic means $\left(\mathrm{n}_{\min }=56, \mathrm{n}_{\max }=71\right)$ 
The fact that existing measures not only need to be continued, but also further elaborated and improved, becomes even clearer when taking into account the employees' assessment of the statement "There are still major cultural differences" (Figure 5): A total of 57\% of respondents agreed with this statement, $29 \%$ of them strongly, while only $13 \%$ considered cultural differences to have been largely or completely overcome.

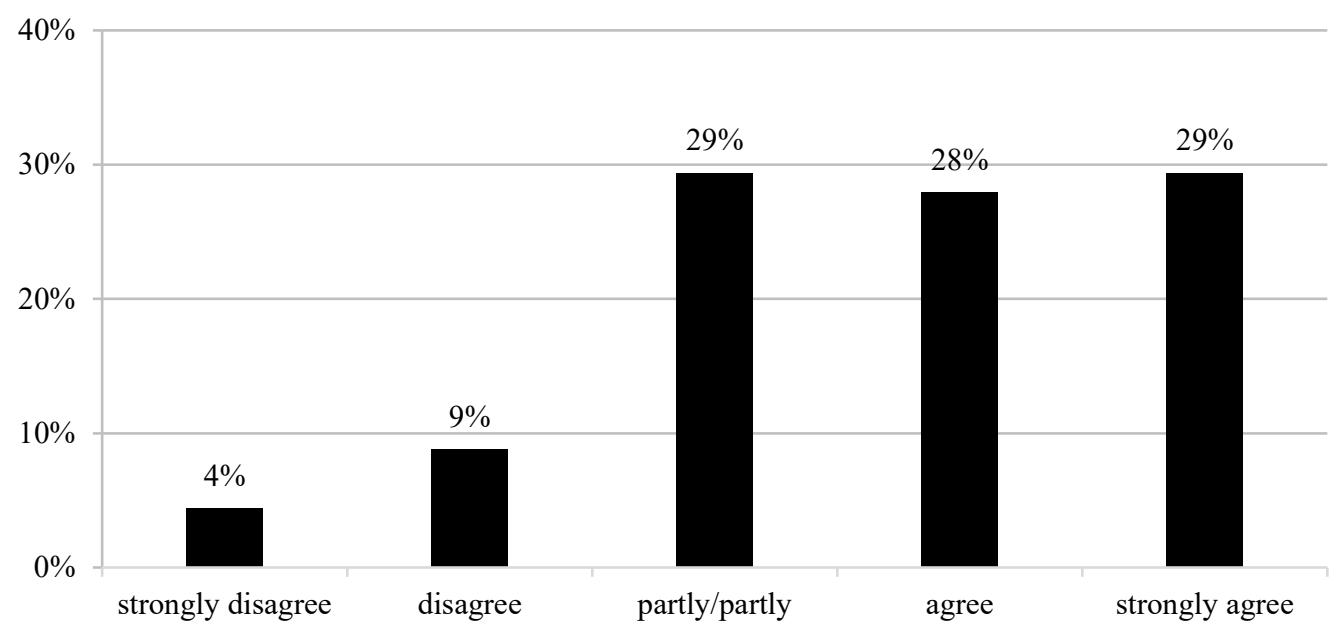

Figure 5: Employees' assessment of the statement "There are still major cultural differences" $(n=68)$

The challenge for the coming years, especially the post-pandemic period, is clearly evident here: Cultural differences, some of which have only become apparent in the closer cooperation during the merger process, must be understood and addressed openly and with seriousness and commitment.

\section{Conclusion}

Managing a university IT merger without disrupting ongoing operations can be considered a challenge in principle. Conducting such a merger during a pandemic, in which the functioning of the entire university depends even more than usual on IT and in which the demands and workload far exceed the usual level, would be a real test for any organisation. The conservative approach to creating a new integrated organisational structure with largely established processes under the umbrella of a joint management has paid off and made WWU IT's rapid responses to the crisis possible in the first place. Difficulties have arisen mainly from the lack of face-to-face teamwork and informal exchange, which are essential for new colleagues to get to know each other and build and strengthen their social cohesion. These aspects can only be inadequately represented by digital formats. At the same time, the pandemic has prompted the creation of new ad-hoc working groups in a very short time as a result of unexpected challenges and has demanded efficient cooperation across departmental boundaries. The positive feedback from stakeholders reflects the joint achievement, which has created a sense of 
community in the context of the merger in a rather unusual way. The following success factors have proven helpful in the WWU IT process:

- regularly informing all employees about the current status and the next steps,

- creating a common identity through a new name, logo, external image and mission statement,

- creating a joint management board at eye level,

- promoting interdepartmental cooperation at the working level,

- establishing feedback channels and prompt addressing of staff concerns, and

- minimising the impact of the merger on services and customer processes.

Nonetheless, the cultural gap is still evident and while efficiency alone may sustain an organisation temporarily, more is needed to ensure durability and generate innovation. Therefore, a management focus should be on testing new measures to strengthen employee satisfaction, spark team spirit and reserve time and space for social and informal exchange - at the latest after the end of the pandemic work mode. Harmonising the working cultures in a way that convinces employees from both original institutions remains the crucial factor for the success of the merger.

\section{References}

Jansen, S. A. (2004). Management von Unternehmenszusammenschlüssen - Theorien, Thesen, Tests und Tools. Stuttgart: Klett-Cotta.

Luik, M. A. (2014). Integrationsmanagement bei Fusionen. Prozessablauf und Strategieentwicklung. Hamburg: Diplomica Verlag GmbH.

Picot, G., \& Picot, M. A. (2012). Wirtschaftliche und wirtschaftsrechtliche Aspekte bei der Planung der Mergers \& Acquisitions. In G. Picot, Handbuch Mergers \& Acquisitions - Planung, Durchführung, Integration (pp. 2-47). Stuttgart: Schäffer-Poeschel. 